\title{
Tractografia: definição e aplicação na ressecção de tumores cerebrais
}

\author{
Eduardo S. Carvalhal Ribas ${ }^{1}$, Manoel J. Teixeira²
}

\section{RESUMO}

O tratamento cirúrgico dos tumores cerebrais envolve a ressecção máxima da lesão, levando a maior chance de controle tumoral e aumento da sobrevida do paciente. Por outro lado, a intervenção cirúrgica deve preservar as áreas eloquentes e minimizar as possíveis sequelas resultantes da cirurgia. Atualmente, há muitos exames disponíveis para auxiliar na programação e estratégia cirúrgica e para atingir esses dois objetivos. Neste artigo, foram analisadas a tractografia e suas aplicações nas cirurgias de tumores cerebrais.

\section{PALAVRAS-CHAVE}

Tractografia, tumores cerebrais.

\section{ABSTRACT}

Tractography: definition and application in brain tumor surgery

Surgical treatment of brain tumors involves the maximum resection of the lesion, leading to a greater chance of tumor control and increase patient survival. On the other hand, surgical intervention should preserve eloquent areas and minimize the possible neurological deficits resulting from the surgery. Currently, many tests are available to assist our surgical planning and strategy, aiming to achieve better results. In this article, we analyze the basic concepts of tractography and its applications in brain tumor surgeries.

\section{KEYWORDS}

Tractography, brain tumors.

\section{Introdução}

Um dos objetivos da neuro-oncologia é a ressecção máxima da lesão tumoral com a preservação máxima do tecido cerebral adjacente, o que, em muitas situações, é de difícil realização para o neurocirurgião. Esse preceito é fundamentalmente importante quando são tratadas lesões em áreas eloquentes, como as relacionadas a linguagem, cognição, funções motoras ou sensoriais. Um grande número de pacientes com tumores em regiões como o córtex motor primário está neurologicamente intacto ou pouco debilitado; nesses casos a distinção entre tecido cerebral intacto e tecido tumoral durante uma cirurgia é crucial para evitar déficits neurológicos durante a ressecção tumoral. ${ }^{1}$

Muitos métodos já foram desenvolvidos com o objetivo de auxiliar o neurocirurgião em sua programação cirúrgica, mostrando os reais limites, a anatomia precisa e a função de cada região. O objetivo final é preservar, ao máximo, as áreas funcionais durante uma interven- ção cirúrgica e aumentar o grau de ressecção tumoral. As ressecções agressivas de neoplasias cerebrais são correlacionadas com aumento da sobrevida e do tempo de preservação funcional do paciente. ${ }^{2,3}$ Alguns neurocirurgiões advogam a teoria de que deve ser ressecada toda região com aparência anormal durante a cirurgia, mantendo-se sob a premissa de que as áreas funcionais estão destruídas ou deslocadas pela neoplasia, porém alguns estudos já demonstraram que pode haver infiltração neoplásica em áreas com manutenção da função dentro do tecido comprometido. ${ }^{4,5}$ A ressecção dessa área que está infiltrada pelo tumor, mas mantém sua função preservada, resulta em déficit neurológico pós-operatório. ${ }^{5}$

A ressonância nuclear magnética (RNM) habitual, a tomografia por emissão de pósitron, a magnetoencefalografia e a RNM funcional são algumas das ferramentas utilizadas na investigação e localização de áreas corticais funcionais. ${ }^{6-10}$ Embora a RNM funcional tenha como finalidade diminuir a necessidade de exames mais in-

1. Médico-residente da Divisão de Clínica Neurocirúrgica do Hospital das Clínicas da Faculdade de Medicina da Universidade de São Paulo (HCFMUSP).

2. Professor titular da Disciplina de Neurocirurgia e Diretor de Clínica Neurocirúrgica do HCFMUSP. 
vasivos como o teste de Wada e o mapeamento cortical intraoperatório, ${ }^{11,12}$ às vezes esses exames confirmatórios são necessários, já tendo sido demonstrado que essa técnica aumenta o grau de ressecção tumoral e diminui a morbidade associada a ressecções agressivas. ${ }^{13} \mathrm{~A}$ RNM habitual tem a capacidade de identificar um tumor cerebral, porém frequentemente não demonstra com precisão os limites da lesão e o grau de envolvimento das fibras nervosas adjacentes. Um hipersinal na pesagem de T2 ou FLAIR ao redor da lesão pode corresponder à infiltração tumoral no tecido cerebral ou simplesmente ao edema vasogênico. ${ }^{1}$

Os resultados obtidos pela RNM funcional são correlacionados fortemente com sinais gerados pela chegada de impulsos e ativação neuronal em uma região cortical. ${ }^{12}$ Por outro lado, esse método nada informa sobre os impulsos que estão viajando em fibras brancas, tornando-o ideal apenas para diminuir o dano funcional relevante à substância cinzenta cortical, e não das fibras subcorticais. ${ }^{14}$

\section{Tractografia}

Recentemente, imagens que medem a tensão da difusão (DTI) são usadas para mapear a rede de fibras nervosas subcortical. ${ }^{15-17}$ A DTI mede a difusão das moléculas de água, descrevendo a movimentação delas no espaço tridimensional e criando um mapa da sua localização. ${ }^{17-19} \mathrm{Em}$ um ambiente em que não há restrições à sua difusão, as moléculas de água movimentam-se livremente em todos os sentidos, formando um mapa sobre a possível localização dessas moléculas sob a forma de uma esfera, isto é, com igual probabilidade de se encontrarem as moléculas de água em todas as direções. O movimento observado é chamado isotrópico (igual em todas as direções). Por outro lado, quando são encontradas barreiras à sua difusão, a movimentação das moléculas de água se faz em uma direção preferencial, uma vez que elas têm mais facilidade em se difundir paralelamente à barreira do que de encontro a ela. ${ }^{6,20}$ Nesse caso, diz-se que o movimento é anisotrópico. Existem vários graus de anisotropia, e essa variação pode ser quantificada por meio de algumas medidas como a "difusibilidade média" (MD) e a "fração de anisotropia" (FA). A primeira mede a magnitude da anisotropia e a segunda mede a direção da anisotropia, sendo o resultado de 0 a 1 , em que 0 indica movimento isotrópico e 1 indica movimento apenas em uma direção, muito anisotrópico. Com a RNM, adquirindo imagens pesadas em difusão com pelo menos seis direções não colineares, é possível estimar a tensão da difusão, sendo a imagem final montada a partir de vetores da FA que indicam a direção de maior difusão das moléculas de água. Erros no registro devidos à movimentação do paciente ou correntes que podem distorcer a imagem podem ser corrigidos por meio de algoritmos durante o registro automático das imagens, ${ }^{21}$ tornando-as mais fidedignas.

No tecido cerebral, as fibras nervosas da substância branca são as barreiras encontradas pelas moléculas de água à sua difusão. A movimentação preferencial da água no sentido paralelo às fibras forma um vetor também paralelo, visto agora como a direção dessas fibras. ${ }^{1,22,23}$ Com essa informação direcional, a organização dos tractos presentes na substância branca pode ser reconstruída por algoritmos de sequenciamento contínuo das fibras e representada usando esquemas de cores, formando imagens conhecidas atualmente como "tractografias" em duas dimensões. ${ }^{16}$ Nessas imagens, as fibras seguem uma codificação de cores atribuída aos eixos x, y e z. Geralmente, são utilizadas as cores: vermelha para a direção direita/esquerda; verde para anterior/posterior; azul para superior/inferior. Deve-se lembrar sempre que os eixos indicam apenas a direção, e não o sentido em que corre a fibra nervosa. Recentemente, alguns grupos desenvolveram técnicas de fazer imagens tridimensional ${ }^{24-26}$ capazes de demonstrar principalmente as fibras grossas desenhadas. Por meio de estudo e conhecimento da rede subcortical de fibras nervosas, pode-se avaliar a relação delas com qualquer lesão cerebral. No caso de lesões que se encontram em apenas um hemisfério, a comparação entre o hemisfério lesado e o hemisfério poupado é de grande ajuda para identificar alterações. Quando as lesões acometem os dois hemisférios, pode ser comparada a imagem do paciente com a de um indivíduo hígido, procurando por divergências entre elas.

\section{Aplicação da tractografia em neoplasias cerebrais}

No caso da avaliação de neoplasias cerebrais, sua localização e anatomia podem ser vistas em uma RNM habitual, muitas vezes até sendo possível saber o tipo e o grau da neoplasia. A tractografia ajuda a melhor compreender a relação da neoplasia com a rede de fibras nervosas subcorticais, podendo elas ser: deslocadas, edemaciadas, infiltradas ou destruídas. ${ }^{1,22,27-29}$ Com mais essa informação, o neurocirurgião consegue planejar melhor sua cirurgia, entendendo os reais limites da lesão, sua proximidade dos principais tractos, além de pesar com mais compreensão o risco-benefício de uma ressecção extensa da neoplasia.

O deslocamento é visto quando a FA é mantida normal, mas a localização da fibra não é anormal quando 
comparada com o hemisfério contralateral ou a orientação delas é anormal no mapa de cores. Na destruição das fibras, a FA está muito reduzida, podendo até impedir que o tracto seja identificado.

A diferenciação entre edema e infiltração é difícil de ser feita. $\mathrm{O}$ edema vasogênico, muito encontrado nas regiões adjacentes às lesões neoplásicas, é visto como hipersinal na pesagem T2 da RNM e reduz a FA causando distorção da tractografia na área edemaciada devido ao acúmulo pronunciado de moléculas de água, ${ }^{1,28,30}$ talvez por a tractografia ser sensível apenas à água extracelular e não medir diretamente os prolongamentos neuronais. ${ }^{31} \mathrm{~A}$ infiltração também leva à redução da FA, tornando a imagem da tractografia muito parecida nos dois casos. Infelizmente, a distinção das duas situações é de grande importância para delimitar de forma precisa a lesão e dar ao neurocirurgião a informação necessária para planejar melhor sua cirurgia.

A diferenciação entre os diferentes tipos de neoplasias pela DTI se mostrou de difícil realização em alguns estudos. ${ }^{32,33}$ Tsuchiya e cols. ${ }^{32}$ partiram da especulação de que poderiam distinguir imagens de pacientes com metástases cerebrais e pacientes com gliomas de baixo grau com a variação da FA, porém seus resultados indicam que não houve diferença significativa da FA entre os dois grupos, sugerindo procurar por outras formas de diferenciação mais fidedignas. Lu e cols. ${ }^{33}$ demonstram em seu estudo que a DM pode ajudar nessa diferenciação, encontrando DM mais altas na área peritumoral de pacientes com metástases cerebrais em comparação com paciente com gliomas, propondo que esse índice seria determinado principalmente pela água extracelular e, em última análise, pelo edema vasogênico. Esse achado não é encontrado de forma consistente em outros estudos. Tropine e cols. ${ }^{34}$ relatam em seu estudo não ter sido encontrada diferença significativa entre os grupos. Por outro lado, ressaltam que o encontro de uma fina camada envolvendo a neoplasia cerebral em que há aumento da FA e diminuição da DM pode corresponder a uma área de maior vascularização ou compressão de fibras brancas, dado que pode auxiliar na delimitação da lesão.

Em outro estudo, Lu e cols. ${ }^{33}$ propuseram um índice para analisar a área peritumoral, chamando-o de índice de infiltração tumoral. Partindo do diagnóstico presumido do tipo de neoplasia por meio da análise de RNM, o estudo separou os pacientes em dois grupos: portadores de gliomas e portadores de meningeomas ou metástases. No primeiro grupo, a região peritumoral é formada por edema e infiltração tumoral, os dois fatores contribuindo para a diminuição da FA; enquanto no segundo grupo a queda da FA é devida apenas a edema, uma vez que tais lesões não infiltram o tecido nervoso. Assim, o índice expressa a diferença entre a FA que seria encontrada se não houvesse infiltração e a FA observada no exame. $\mathrm{O}$ encontro de índice próximo a 0 indicaria uma ausência de infiltração tumoral e números maiores seriam relacionados a lesões infiltrativas como gliomas.

\section{Conclusão}

Os trabalhos encontrados na literatura são, em sua maioria, análises pontuais de pacientes e têm como foco encontrar um índice para diferenciar, basicamente, lesões infiltrativas de não infiltrativas. Esse resultado tem utilidade dupla: auxílio no diagnóstico patológico da lesão e na delimitação real da lesão para programação cirúrgica. Alguns deles conseguem chegar próximo aos seus objetivos, mas no total não afirmam de forma consistente o mesmo resultado, por não atingirem dados estatisticamente significativos.

Ainda faltam estudos com número maior de pacientes e desenhados com o objetivo de determinar a real vantagem desse exame sobre os outros disponíveis. Se a tractografia melhora significativamente o planejamento cirúrgico, levando a maior grau de ressecção tumoral com preservação funcional do paciente, ainda é uma questão em aberto, mas que deve ser respondida em um futuro próximo.

\section{Referências}

1. Witwer BP, Moftakhar R, Hasan KM, Deshmukh P, Haughton V, Field A, et al. Diffusion-tensor imaging of white matter tracts in patients with cerebral neoplasm. J Neurosurg. 2002;97:568-75.

2. Ammirati M, Vick N, Liao YL, Ciric I, Mikhael M. Effect of the extent of surgical resection on survival and quality of life in patients with supratentorial glioblastomas and anaplastic astrocytomas. Neurosurgery. 1987;21:201-6.

3. Ciric I, Ammirati M, Vick N, Mikhael M. Supratentorial gliomas: surgical considerations and immediate postoperative results. Gross total resection versus partial resection. Neurosurgery. 1987;21:21-6.

4. Ojemann JG, Miller JW, Silbergeld DL. Preserved function in brain invaded by tumor. Neurosurgery. 1996;39:253-9.

5. Skirboll SS, Ojemann GA, Berger MS, Lettich E, Winn HR. Functional cortex and subcortical white matter located within gliomas. Neurosurgery. 1996;38:678-85.

6. Atlas SW, Howard RS II, Maldjian J, Alsop D, Detre $\mathrm{JA}$, Listerud J, et al. Functional magnetic resonance imaging of regional brain activity in patients with intracerebral gliomas: findings and implications for clinical management. Neurosurgery. 1996;38:329-38.

7. Bittar RG, Olivier A, Sadikot AF, Andermann F, Reutens DC. Cortical motor and somatosensory representation: effect of cerebral lesions. J Neurosurg. 2000;92:242-8.

8. Grafton ST, Woods RP, Mazziotta JC, Phelps ME. Somatotopic mapping of the primary motor cortex in humans: activation studies with cerebral blood flow 
and positron emission tomography. J Neurophysiol. 1991;66:735-43.

9. Lehericy S, Duffau H, Cornu P, Capelle L, Pidoux B, Carpentier A, et al. Correspondence between functional magnetic resonance imaging somatotopy and individual brain anatomy of the central region: comparison with intraoperative stimulation in patients with brain tumors. J Neurosurg. 2000;92:589-98.

10. McDonald JD, Chong BW, Lewine JD, Jones G, Burr $\mathrm{RB}, \mathrm{McDonald} \mathrm{PR}$, et al. Integration of preoperative and intraoperative functional brain mapping in a frameless stereotactic environment for lesions near eloquent cortex. Technical note. J Neurosurg. 1999;90:591-8.

11. Lundquist $P$, Backlund EO, Sjoqvist $L$, Thoumas KA, Wigstrom L, Brismar T. Clinical application of functional magnetic resonance imaging (fMRI) to surgery in the brain. J Neuroimaging. 1997;7(2):131-3.

12. Tomczak RJ, Wunderlich AP, Wang $Y$, Braun V, Antoniadis G, Gorich J, et al. fMRI for preoperative neurosurgical mapping of motor cortex and language in a clinical setting. J Comput Assist Tomogr. 2000;24(6):927-34.

13. Berger MS, Ojemann GA. Intraoperative brain mapping techniques in neuro-oncology. Stereotact Funct Neurosurg. 1992;58:153-61.

14. Hendler T, Pianka P, Sigal M, Kafri M, Ben-Bashat D, Constantini $S$, et al. Delineating gray and white matter involvement in brain lesions: three-dimensional alignment of functional magnetic resonance and diffusion-tensor imaging. J Neurosurg. 2003;99(6):1018-27.

15. Doran M, Hajnal JV, Van Bruggen N, King MD, Young IR, Bydder GM. Normal and abnormal white matter tracts shown by MR imaging using directional diffusion weighted sequences. J Comput Assist Tomogr. 1990;14:865-73.

16. Pajevic S, Pierpaoli C. Color schemes to represent the orientation of anisotropic tissues from diffusion tensor data: application to white matter fiber tract mapping in the human brain. Magn Reson Med. 1999;42:526-40.

17. Pierpaoli C, Jezzard P, Basser PJ, Barnett A, Di Chiro G. Diffusion tensor MR imaging of the human brain. Radiology. 1996;201:637-48.

18. Brunberg JA, Chenevert TL, McKeever PE, Ross DA, Junck $\mathrm{LR}$, Muraszko KM, et al. In vivo MR determination of water diffusion coefficients and diffusion anisotropy: correlation with structural alteration in gliomas of the cerebral hemispheres. AJNR Am J Neuroradiol. 1995;16(2):361-71.

19. Chien D, Kwong KK, Gress DR, Buonanno FS, Buxton $\mathrm{RB}$, Rosen BR. MR diffusion imaging of cerebral infarction in humans. AJNR Am J Neuroradiol. 1992;13:1097-105.

20. Jones DK, Simmons A, Williams SC, Horsfield MA. Noninvasive assessment of axonal fiber connectivity in the human brain via diffusion tensor MRI. Magn Reson Med. 1999;42:37-41.

21. Woods RP, Mazziotta JC, Cherry SR. MRI-PET registration with automated algorithm. J Comput Assist Tomogr. 1993;17:536-46.

22. Basser PJ, Pierpaoli C. A simplified method to measure the diffusion tensor from seven MR images. Magn Reson Med. 1998;39(6):928-34.
23. Logothetis NK, Pauls J, Augath M, Trinath T, Oeltermann A. Neurophysiological investigation of the basis of the fMR signal. Nature. 2001;412(6843):150-7.

24. Basser PJ, Pajevic S, Pierpaoli C, Duda J, Aldroubi A. In vivo fiber tractography using DT-MRI data. Magn Reson Med. 2000;44:625-32.

25. Conturo TE, Lori NF, Cull TS, Akbudak E, Snyder AZ, Shimony JS, et al. Tracking neuronal fiber pathways in the living human brain. Proc Nat Acad Sci USA. 1999;96:10422-7.

26. Mori S, Frederiksen K, Van Zijl PC, Stieltjes B, Kraut MA, Solaiyappan $\mathrm{M}$, et al. Brain white matter anatomy of tumor patients evaluated with diffusion tensor imaging. Ann Neurol. 2002;51(3):377-80.

27. Holodny Al, Ollenschleger MD, Liu WC, Schulder M, Kalnin AJ. Identification of the corticospinal tracts achieved using blood-oxygen-level-dependent and diffusion functional MR imaging in patients with brain tumors. AJNR Am J Neuroradiol. 2001;22(1):83-8.

28. Sinha S, Bastin ME, Whittle IR, Wardlaw JM. Diffusion tensor MR imaging of high-grade cerebral gliomas. AJNR Am J Neuroradiol. 2002;23(4):520-7.

29. Wieshmann UC, Krakow K, Symms MR, Parker GJ, Clark CA, Barker GJ, et al. Combined functional magnetic resonance imaging and diffusion tensor imaging demonstrate widespread modified organization in malformation of cortical development. J Neurol Neurosurg Psychiatry. 2001;70(4):521-3.

30. Werring DJ, Toosy AT, Clark CA, Parker GJ, Barker GJ, Miller $\mathrm{DH}$, et al. Diffusion tensor imaging can detect and quantify corticospinal tract degeneration after stroke. $J$ Neurol Neurosurg Psychiatry. 2000;69(2):269-72.

31. Kraemer F, Darquie A, Clark CA, Le Bihan D. Separation of two diffusion compartments in the human brain. Proc Intl Soc Magn Reson Med. 1999;7:1808.

32. Tsuchiya K, Fujikawa A, Nakajima M, Honya K. Differentiation between solitary brain metastasis and high-grade glioma by diffusion tensor imaging. Br J Radiol. 2005;78:533-7.

33. Lu S, Ahn D, Johnson G, Cha S. Peritumoral diffusion tensor imaging of high-grade gliomas and metastatic brain tumors. AJNR Am J Neuroradiol. 2003;24:937-41.

34. Tropine A, Vucurevic G, Delani MS, Boor S, Hopf N, Bohl J, et al. Contribution of diffusion tensor imaging to delineation of gliomas and glioblastomas. J Magn Reson Imaging. 2004;20:905-12.

35. Stanley L, Daniel A, Glyn Johnson BS, Meng L, David Z, Grossman RI. Diffusion-tensor MR imaging of intracranial neoplasia and associated peritumoral edema: introduction of the tumor infiltration index. Radiology. 2004;232:221-8.

\section{Endereço para correspondência}

Eduardo Santamaria Carvalhal Ribas

Rua Prof. Eduardo Monteiro, 567, Morumbi.

05614-120 - São Paulo, SP, Brasil

Email: eribas@gmail.com 